\title{
Narrative Review of Web-based Healthy Lifestyle Interventions for Cancer Survivors
}

\author{
Victoria Williams ${ }^{1 *}$, Nashira Brown ${ }^{1}$, Alahni Becks ${ }^{2}$, Dori Pekmezi ${ }^{1}$ and Wendy Demark-Wahnefried ${ }^{4}$ \\ ${ }^{1}$ Department of Health Behavior, University of Alabama at Birmingham (UAB), Birmingham, Alabama, USA \\ ${ }^{2}$ Tuskegee University, Tuskegee, Alabama, USA
}

${ }^{3}$ Department of Nutritional Sciences, UAB, Birmingham, Alabama, USA

${ }^{4} U A B$ Comprehensive Cancer Center, Birmingham, Alabama, USA

Submission: February 18, 2020; Published: March 10, 2020

"Corresponding author: Victoria Williams, Department of Health Behavior, University of Alabama at Birmingham (UAB), Birmingham, Alabama, USA

\begin{abstract}
This narrative review of web-delivered weight management, diet quality, and physical activity interventions for cancer survivors relies on a systematic search of PubMed, Psych Info, and EBSCOhost which identified 19 unique web-delivered lifestyle interventions for cancer survivors. The sample sizes for these studies ranged from 11-492. Intervention duration ranged from 1-12 months; however, most interventions were 6-12 weeks in length. Ten studies were randomized controlled trials (RCTs), two were two-arm quasi RCTs, and seven employed a single-arm pre/post-test design. Many $(\mathrm{N}=15)$ of the interventions were well-grounded in behavioral theory, which may have led to favorable behavior change. Most studies (15-of-19) targeted and reported increases in physical activity, while only a few targeted and reported improvements in diet quality ( $36.9 \%$ and $15.8 \%$, respectively) and weight management ( $26.3 \%$ and $10.5 \%$, respectively). A notable limitation was that most studies were conducted among populations that were primarily White and female. Future directions for Internet-based lifestyle interventions for cancer survivors include increasing: (a) focus on multiple behavior change, (b) representation of male and minority populations to improve generalizability of findings, (c) extended intervention duration and follow-up to evaluate long-term efficacy of web-based lifestyle interventions, and (d) sample size to allow for adequate statistical power.

Keywords: Cancer survivors; Web-based; Lifestyle intervention; Nutrition; Physical activity

Abbreviations: PRISMA: Preferred Reporting Items for Systematic Reviews and Meta-Analyses; DQI : Diet Quality Index; SQUASH: Short Questionnaire to Assess Health-Enhancing; PAR: Physical Activity Recall; SQUASH: Short Questionnaire to Assess Health-Enhancing; IPAQ: International Physical Activity Questionnaires; CAPS: Cross-Cultural Activity Participation Study; METs: Metabolic Equivalents; SCT: Social Cognitive Theory; TTM: Transtheoretical Model; TTM: Transtheoretical Model; TPB: Theory of Planned Behavior; HBM: Health Belief Model; PAPM: Precaution Adoption Process Model.
\end{abstract}

\section{Introduction}

By the year 2029, there will be an estimated 21.7 million cancer survivors [1]; a population increasingly growing due to the aging population, improved early detection, and modern therapeutics and treatment modalities [1]. However, cancer survivors represent a vulnerable population characterized by high rates of obesity, physical inactivity, poor diets, and related chronic conditions [2-4]. Face-to-face lifestyle interventions have shown promise for improving the quality-of-life and reducing adverse health outcomes among cancer survivors [5-8]. However, these approaches are relatively costly and thus have limited reach to the survivors who need them most [8]. Web-delivered lifestyle interventions have already shown success in other populations $[9,10]$ and have the potential to reach large numbers of cancer survivors at relatively low cost. Moreover, web-based strategies can overcome obstacles cited by cancer survivors by not requiring transportation or clinic visits [8]. In fact, they can be accessed 24/7 from any Internet-enabled device [11-13]. Past studies indicate that cancer survivors are already using the Internet to obtain healthy lifestyle information [14] and report a lack of credible online resources for healthy lifestyle behavior change information [15]. Thus, there is an evident interest and need for web-based lifestyle intervention in this patient population.

Findings-to-date from studies in this area have been encouraging. Prior related reviews on non-face-to-face lifestyle interventions (including only three web-based interventions) [16], eHealth physical activity interventions [17], and digital diet and physical activity interventions for cancer survivors [18] 
all found the number of health behavior change interventions are increasing. It is important to note that digital and eHealth interventions are distinct from web-based interventions. Webbased interventions require an Internet connection for program delivery, whereas eHealth interventions use the Internet specifically to provide healthcare services, while digital programs do not require the Internet for intervention delivery (e.g., Gaming systems like Wii can be operated by inserting a game into a console without an Internet connection). Nevertheless, there has yet to be a review of web-based physical activity and diet interventions (as both behaviors are critical to maintaining a healthy weight) for cancer survivors. Therefore, there is a need for a review of web-based lifestyle intervention research in cancer survivors to help identify the next steps and move this field forward, given the critical public health implications and rapidly evolving nature of the Internet.

\section{Methods}

\section{Study Design}

The guidelines of the "Preferred Reporting Items for Systematic Reviews and Meta-Analyses" (PRISMA) [19] were followed to systematically search the literature to identify studies examining web-delivered lifestyle interventions for cancer survivors. The search was conducted for papers published through August 10, 2019.

\section{Study Selection}

PubMed, Psych Info, and EBSCOhost were systematically searched for articles published on web-based nutrition, physical activity, and/or weight management interventions for adult cancer survivors. The research intersected terms for physical activity (exercise), weight (weight loss, weight management, weight change, weight reduction), nutrition (diet), Internet (web, web-based), cancer (cancer survivorship, tumor, neoplasm) and interventions (programs). Duplicate papers, papers not written in English, those containing non-human and non-adult samples were manually removed through the study screening process. Included studies met the following criteria: web- or Internet-based programs requiring an Internet connection to access intervention materials (e.g., sending or receiving an email, visiting a website or accessing a mobile application) and targeting weight management, physical activity, and/or nutrition in adult cancer survivors diagnosed with any cancer type during or post-treatment.

Studies were excluded for the following reasons: a) study was not exclusively for cancer survivors; b) non-web or Internetbased studies; c) not in English; d) only available as an abstract; d) overlapping publications; e) protocol papers, and f) review papers. Intervention studies with quasi-experimental and pre-/ post-test research designs were included as this is an emerging field. Those early studies may help shed light on future directions and provide a holistic view of current research. Two researchers independently identified and reviewed studies potentially meeting eligibility criteria. First, the titles and abstracts were reviewed, and duplicate articles were removed. For studies passing the initial screening, the article's full text was reviewed. A data extraction form was used to record pertinent information on each study consistently. The form included sections on the papers' authors, country of origin, publication year, study design, cancer type, intervention activities, number of participants, intervention duration, and behavioral outcomes. Any discrepancies were resolved by consensus or through discussion with a third researcher.

\section{Results}

See Figure 1 for the PRISMA flow diagram of the study selection process. The search strategy initially identified 862 records; 19 unique web-based lifestyle interventions for cancer survivors met the inclusion criteria and were included in this review. The included articles were published between 2011 and 2018. Table 1 provides a summary of each of the studies included in the review.

Participant characteristics: The number of study participants ranged from 11 to 492 cancer survivors. Fourteen studies [20-33] had a sample size less than 100, and six studies [24,25,27,30-32] enrolled 49 participants or less. Participants mean age ranged from 23 to 73.2 years of age. Three interventions were conducted specifically among young adult cancer survivors [20,25,31], one of which was for young adult cancer survivors diagnosed in childhood [31]. Another study was explicitly for older adult cancer survivors [27]. Six countries were represented in the review. Most studies $(\mathrm{N}=9)$ were conducted in the United States $[20,21,25$ $27,29-31,34]$, followed by the Netherlands $(N=3)[33,35,36]$, Australia $(\mathrm{N}=2)[23,37]$, and South Korea $(\mathrm{N}=2)[28,38]$. Other countries represented in the review include Canada [22,24], and the United Kingdom [32].

Most studies ( $\mathrm{N}=12)$ included both male and female cancer survivors [20,22,23,25,27,30-36]. However, breast cancer survivors were over-represented in these samples. Six studies were exclusively for breast cancer survivors (100\% female) $[21,26,28,33,37,38]$, one study was for breast and endometrial cancer survivors (100\% female) [29], two for breast, colorectal, and prostate cancer survivors (56\% and $82 \%$ female) [22,32], and another with testicular and breast cancer survivors (65\% female) [30]. Additionally, prostate cancer survivors were also targeted as there was one study exclusively for prostate cancer survivors (100\% male) [24], and another with colorectal and prostate cancer survivors (87\% male) [36]. As for minority representation, most participants in these studies were White. Only one study included a substantial number of racial and ethnic minorities (83\% African Americans, 11\% Hispanics, and 6\% mixed population) [26]. Other studies $(\mathrm{N}=6)$ did not describe the ethnic/racial makeup of their population [28,33,35-38].

Study design, duration and attrition: Of the 19 studies, 10 were randomized controlled trials (RCTs) with two [20-22,25,28, 34-36] or three arms [37]. Two more were two-arm quasi RCTs 
$[23,38]$ due to lack of blinding [23] and randomization practices (participants were assigned based on whether they owned a smartphone) [38]. Seven studies had a single arm pre/post-test design [24,27,29-33].

Table 1: Studies included in narrative review of web-based lifestyle interventions for cancer survivors.

\begin{tabular}{|c|c|c|c|c|c|c|c|c|}
\hline $\begin{array}{c}\text { First } \\
\text { author, } \\
\text { year [ref] } \\
\text { Country }\end{array}$ & $\begin{array}{c}\text { Baseline } \\
\text { Sample } \\
\text { Character- } \\
\text { istics }\end{array}$ & $\begin{array}{c}\text { Delivery } \\
\text { Format }\end{array}$ & $\begin{array}{l}\text { Intervention De- } \\
\text { scription }\end{array}$ & $\begin{array}{c}\text { Behav- } \\
\text { ior(s) } \\
\text { Targeted }\end{array}$ & $\begin{array}{c}\text { Intervention } \\
\text { Duration/ } \\
\text { Follow-up }\end{array}$ & $\begin{array}{c}\text { Theoreti- } \\
\text { cal Frame- } \\
\text { work }\end{array}$ & $\begin{array}{c}\text { Mea- } \\
\text { sure(s) }\end{array}$ & Major Findings \\
\hline $\begin{array}{l}\text { Rabin, } \\
2011 \text { [1] } \\
\text { USA }\end{array}$ & $\begin{array}{c}\text { Sedentary } \\
\text { young adult } \\
\text { cancer survi- } \\
\text { vors } \\
\text { N=18/mean } \\
\text { age }=32 \\
\text { years } / 56 \% \\
\text { female } / 94 \% \\
\text { White }\end{array}$ & Website & $\begin{array}{l}\text { 2-arm RCT } \\
\text { Access to a physical } \\
\text { activity website for } \\
\text { cancer survivors, } \\
\text { Step in Motion. Set } \\
\text { PA goals and log } \\
\text { Control was given } \\
\text { info about three can- } \\
\text { cer survivor websites } \\
\text { that did not provide } \\
\text { info about PA. }\end{array}$ & $\mathrm{PA}$ & 12 weeks & $\begin{array}{l}\text { Transthe- } \\
\text { oretical } \\
\text { Model } \\
\text { Social } \\
\text { Cognitive } \\
\text { Theory }\end{array}$ & PA: PAR & $\begin{array}{c}\text { Increased PA } \\
\text { Baseline: } \\
58.75 \text { mins/week (44.54) vs. } \\
39.00 \text { (35.65), p=0.078 } \\
\text { Follow-up: } \\
161.25 \text { (221.79) vs. } 55.50 \\
\text { (77.48), p=0.48 } \\
\text { Attrition } \\
\text { Intervention: } 5.6 \% \text { at fol- } \\
\text { low-up } \\
\text { Control: } 0 \% \text { at follow-up }\end{array}$ \\
\hline $\begin{array}{c}\text { Hatch- } \\
\text { ett,2013 } \\
\text { [2] } \\
\text { USA }\end{array}$ & $\begin{array}{c}\text { Sedentary } \\
\text { breast cancer } \\
\text { survivors } \\
\mathrm{N}=85 / \text { mean } \\
\text { age }=\text { not re- } \\
\text { ported/ aged } \\
18+/ 100 \% \\
\text { female/95\% } \\
\text { White }\end{array}$ & Email & $\begin{array}{c}\text { 2-arm RCT } \\
\text { Eight e-mails over } 12 \\
\text { weeks } \\
\text { Control group } \\
\text { offered } \\
\text { intervention after } \\
\text { end of the trial }\end{array}$ & $\mathrm{PA}$ & $\begin{array}{c}12 \text { weeks with } \\
\text { measurements } \\
\text { at } 6 \text { and } 12 \\
\text { weeks }\end{array}$ & $\begin{array}{c}\text { Social } \\
\text { Cognitive } \\
\text { Theory }\end{array}$ & $\begin{array}{l}\text { PA: } \\
\text { PAR }\end{array}$ & $\begin{array}{c}\text { Increased PA } \\
\text { Baseline: } \\
0 \text { days vs. } 0 \text { days } \\
\text { Follow-up: } \\
3.47 \text { days (2.19) vs. } 1.42 \text { days } \\
\text { (1.67), p<0.001 } \\
\text { Attrition } \\
\text { Overall: } 12.9 \% \text { at follow-up } \\
\text { Intervention: } 11.6 \% \text { at fol- } \\
\text { low-up } \\
\text { Control: } 14.3 \% \text { at follow-up }\end{array}$ \\
\hline $\begin{array}{c}\text { Valle, } \\
2013 \text { [3] } \\
\text { USA }\end{array}$ & $\begin{array}{c}\text { Young adult } \\
\text { cancer survi- } \\
\text { vors } \\
\text { N=86/mean } \\
\text { age }=32 \\
\text { years } / 91 \% \\
\text { female } / 91 \% \\
\text { White }\end{array}$ & $\begin{array}{l}\text { Website } \\
\text { and Face- } \\
\text { book }\end{array}$ & $\begin{array}{l}\text { 2-arm RCT: FITNET } \\
\text { or SC (self-help com- } \\
\text { parison) } \\
\text { FITNET: Facebook } \\
\text { group and study } \\
\text { website access. Pro- } \\
\text { vides tools for goal } \\
\text { setting, PA feedback, } \\
\text { reminders to log PA } \\
\text { SC: Assigned } \\
\text { different Facebook } \\
\text { group that provides } \\
\text { information and } \\
\text { weekly private mes- } \\
\text { sages with basic PA } \\
\text { information. }\end{array}$ & $\mathrm{PA}$ & 12 weeks & $\begin{array}{c}\text { Social } \\
\text { Cognitive } \\
\text { Theory }\end{array}$ & PA: GLTEQ & $\begin{array}{c}\text { Increased PA } \\
\text { Fit NET: } \\
187.6 \text { mins/week (171.1) vs. } \\
397.7 \text { (778.4), p=0.009 } \\
\text { Control: } \\
199.3 \text { mins/week }(151.5) \text { vs. } \\
265.9 \text { (228.1),p= } 0.045 \\
\text { Attrition } \\
\text { Overall: } 18.6 \% \text { at follow-up } \\
\text { FITNET: } 22.2 \% \text { at follow up } \\
\text { SC: } 14.6 \% \text { at follow-up }\end{array}$ \\
\hline $\begin{array}{c}\text { Bantum, } \\
2014 \text { [4] } \\
\text { USA }\end{array}$ & $\begin{array}{l}\text { Breast, } \\
\text { colorectal, } \\
\text { endometri- } \\
\text { um/uterine/ } \\
\text { ovarian, } \\
\text { non-Hod- } \\
\text { gkin's lym- } \\
\text { phoma, lung, } \\
\text { thyroid, and } \\
\text { oral cancer } \\
\text { survivors } \\
\text { N= 352/ } \\
\text { mean age = } \\
51 \text { years/ } \\
\text { 82.1\% fe- } \\
\text { male/ 87.2\% } \\
\text { White }\end{array}$ & Website & $\begin{array}{c}\text { Two-arm de- } \\
\text { layed-treatment RCT } \\
\text { Online workshop } \\
\text { (website) to encour- } \\
\text { age healthy lifestyle } \\
\text { behaviors }\end{array}$ & $\begin{array}{l}\text { PA } \\
\text { Diet } \\
\text { Depres- } \\
\text { sion } \\
\text { Fatigue }\end{array}$ & $\begin{array}{l}6 \text { weeks with } \\
6 \text {-month } \\
\text { follow-up }\end{array}$ & $\mathrm{N} / \mathrm{A}$ & $\begin{array}{l}\text { PA: GLTEQ } \\
\text { Diet: Block } \\
\text { Food } \\
\text { Frequency } \\
\text { Question- } \\
\text { naire }\end{array}$ & $\begin{array}{c}\text { Increased PA } \\
\text { Intervention: } \\
106 \text { mins/week vs. } 137 \\
\text { Control: } \\
86.0 \text { mins/week vs. } 96.2 \\
\text { No change in diet } \\
\text { Attrition } \\
\text { Overall: } 13.9 \% \text { at 6-month } \\
\text { follow-up } \\
\text { Intervention: } 16.5 \% \text { at 6- } \\
\text { month follow-up } \\
\text { Control: } 11.4 \% \text { at } 6 \text { month } \\
\text { follow-up }\end{array}$ \\
\hline
\end{tabular}




\begin{tabular}{|c|c|c|c|c|c|c|c|c|}
\hline $\begin{array}{c}\text { Berg, } \\
2014 \text { [5] } \\
\text { USA }\end{array}$ & $\begin{array}{c}\text { Young adults' } \\
\text { survivors of } \\
\text { childhood } \\
\text { cancer } \\
\mathrm{N}=24 / \text { mean } \\
\text { age }=23 / 71 \\
\% \text { female/ } \\
83 \% \text { White }\end{array}$ & $\begin{array}{c}\text { Website } \\
\text { and email }\end{array}$ & $\begin{array}{l}\text { One-arm, pre/post- } \\
\text { test design Website \& } \\
12 \text { modules delivered } \\
\text { via email bi-weekly } \\
\text { Tailored feedback }\end{array}$ & $\begin{array}{l}\text { PA } \\
\text { Reducing } \\
\text { alcohol/ } \\
\text { cigarette } \\
\quad \text { use }\end{array}$ & 6 weeks & $\begin{array}{l}\text { Theory of } \\
\text { Reasoned } \\
\text { Action }\end{array}$ & $\begin{array}{l}\text { study } \\
\text { question- } \\
\text { naire }\end{array}$ & $\begin{array}{l}\text { No significant health behavior } \\
\text { changes } \\
\text { Attrition } \\
4.2 \% \text { at week } 6 ; 20.8 \% \text { at } \\
\text { follow up }\end{array}$ \\
\hline $\begin{array}{c}\text { Frensham, } \\
2014 \text { [6] } \\
\text { Frensham, } \\
2014 \text { [7] } \\
\text { Frensham, } \\
2018 \text { [8] } \\
\text { Frensham, } \\
2018 \text { [9] } \\
\text { Australia }\end{array}$ & $\begin{array}{c}\text { Sedentary } \\
\text { adult cancer } \\
\text { survivors } \\
\text { N=91 mean } \\
\text { age }=66 \\
\text { years } / 52 \% \\
\text { female/ } 96 \% \\
\text { White }\end{array}$ & Website & $\begin{array}{l}\text { 2-arm quasi-RCT } \\
2 \text { groups: Interven- } \\
\text { tion and wait-list } \\
\text { control } \\
\text { Intervention: STRIDE } \\
\text { website and weekly } \\
\text { step goals. Website } \\
\text { provides info on } \\
\text { healthy lifestyles for } \\
\text { cancer survivors }\end{array}$ & $\begin{array}{l}\text { PA } \\
\text { Second- } \\
\text { ary: } \\
\text { Weight } \\
\text { manage- } \\
\text { ment }\end{array}$ & $\begin{array}{l}12 \text { weeks with } \\
3 \text {-month } \\
\text { follow-up }\end{array}$ & $\begin{array}{c}\text { Social } \\
\text { Cognitive } \\
\text { Theory }\end{array}$ & $\begin{array}{c}\text { PA: } \\
\text { New-Life- } \\
\text { styles } \\
\text { Pedometer }\end{array}$ & $\begin{array}{c}\text { Increased PA after inter- } \\
\text { vention (week 12) with no } \\
\text { changes after follow-up } \\
\text { Baseline-STRIDE vs control: } \\
7055 \text { steps/day (2633) vs } \\
6667 \text { steps/day (2993); }=.56 \\
\text { Week 12:STRIDE vs control: } \\
9274 \text { steps/day (3579) vs } \\
7499 \text { steps/day (3320) p= .04 } \\
\text { Follow-up: STRIDE vs control: } \\
8437 \text { steps/day ( } 3487 \text { ) vs } \\
7333 \text { steps/day ( } 4044 \text { ) p=.55 } \\
\text { Attrition } \\
\text { Overall: } \\
\text { 18.6\% at } 12 \text { weeks; } 18.6 \% \text { at } 3 \\
\text { month follow up } \\
\text { Intervention: } 23.5 \% \text { at } 12 \\
\text { weeks; } 23.5 \% \text { at } 3 \text { months } \\
\text { Control: } 13.7 \% \text { at } 12 \text { weeks; } \\
13.7 \% \text { at } 3 \text { months }\end{array}$ \\
\hline $\begin{array}{c}\text { Lee, } 2014 \\
{[10]} \\
\text { South } \\
\text { Korea }\end{array}$ & $\begin{array}{l}\text { Breast cancer } \\
\text { survivors } \\
\text { N=59/ mean } \\
\text { age }=42 \\
\text { years } / 100 \% \\
\text { female }\end{array}$ & Website & $\begin{array}{l}\text { Two-arm RCT: WSE- } \\
\text { DI vs control } \\
\text { WSEDI: website } \\
\text { containing tailored } \\
\text { information on goal } \\
\text { progress, action } \\
\text { planning, goal setting } \\
\text { and automatic } \\
\text { feedback } \\
\text { Control: } 50 \text {-page } \\
\text { educational booklet } \\
\text { on diet and exercise }\end{array}$ & $\begin{array}{l}\text { PA } \\
\text { Diet }\end{array}$ & 12 weeks & $\begin{array}{c}\text { Transthe- } \\
\text { oretical } \\
\text { Model }\end{array}$ & $\begin{array}{l}\text { PA: 7-day } \\
\text { diaries, } \\
\text { study gen- } \\
\text { erated } \\
\text { Diet: 3-day } \\
\text { diet recall, } \\
\text { diet qual- } \\
\text { ity index } \\
\text { (DQI) }\end{array}$ & $\begin{array}{c}\text { Increased PA } \\
\text { Baseline-- WSEDI vs control: } \\
10 \text { (33.3) vs. } 10 \text { ( } 34.5) \\
\text { Follow-up-- WSEDI vs control: } \\
19 \text { (65.5) vs. } 10 \text { (35.7), } \\
\text { p<0.0001 } \\
\text { Increased vegetable \& fruit } \\
\text { intake } \\
\text { Improved diet quality } \\
\text { Attrition } \\
\text { Overall: } 3.4 \% \text { at follow-up } \\
\text { WSEDI: } 3.4 \% \text { at follow-up } \\
\text { Control: } 3.3 \% \text { at follow-up }\end{array}$ \\
\hline $\begin{array}{l}\text { Forbes, } \\
2015[11] \\
\text { Forbes, } \\
2017[12] \\
\text { Canada }\end{array}$ & $\begin{array}{c}\text { Breast, } \\
\text { colorectal, } \\
\text { and prostate } \\
\text { cancer survi- } \\
\text { vors } \\
\mathrm{N}=95 / \text { mean } \\
\text { age }=65.1 \\
\text { years } / 56 \% \\
\text { female } / 99 \% \\
\text { White }\end{array}$ & $\begin{array}{c}\text { Website } \\
\text { (online } \\
\text { workshop) } \\
\text { and emails }\end{array}$ & $\begin{array}{l}\text { 2-arm RCT } \\
\text { UCAN: } 9 \text { module } \\
\text { behavior change } \\
\text { program using the } \\
\text { UWALK website to } \\
\text { track their PA and } \\
\text { weekly emails } \\
\text { Control group re- } \\
\text { ceived usual care (no } \\
\text { intervention). }\end{array}$ & PA & 9 weeks & $\begin{array}{l}\text { Theory of } \\
\text { Planned } \\
\text { Behavior }\end{array}$ & PA: GLTEQ & $\begin{array}{c}\text { Increased PA } \\
\text { Baseline-UCAN vs. control: } \\
231 \text { (269) vs. } 212 \text { (216) } \\
\text { Follow-up-UCAN vs. control: } \\
294 \text { (354) vs. } 241 \text { (197) } \\
\text { Attrition } \\
\text { Overall: } 11.6 \% \text { at } 9 \text { weeks } \\
\text { follow-up } \\
\text { UCAN: } 14.6 \% \text { at } 9 \text { weeks } \\
\text { follow-up } \\
\text { control: } 8.5 \% \text { at } 9 \text { weeks } \\
\text { follow-up }\end{array}$ \\
\hline
\end{tabular}




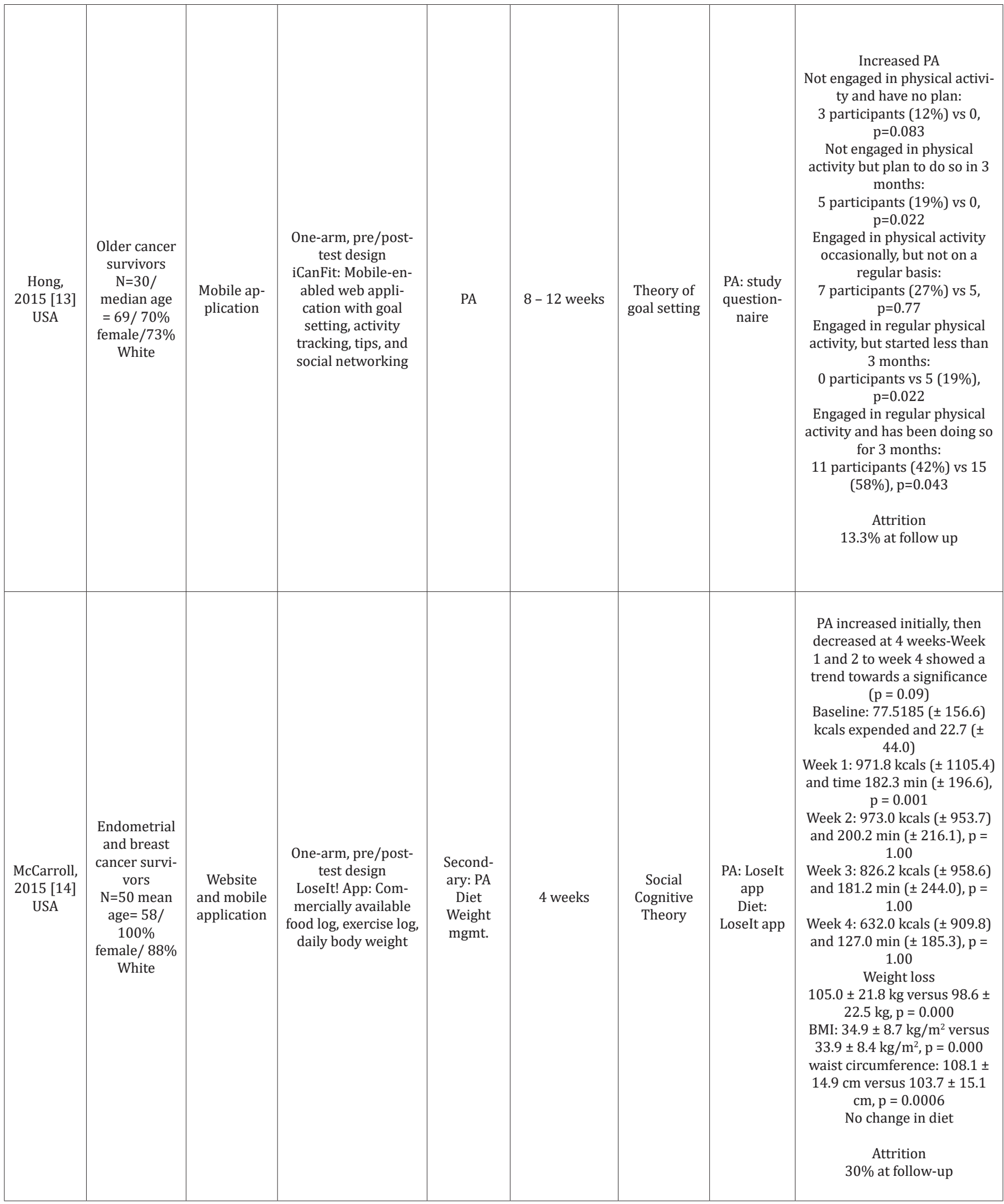




\begin{tabular}{|c|c|c|c|c|c|c|c|c|}
\hline $\begin{array}{l}\text { Kanera, } \\
2016[15] \\
\text { Kanera, } \\
2016[16] \\
\text { Kanera, } \\
2017[17] \\
\text { Nether- } \\
\text { lands }\end{array}$ & $\begin{array}{l}\text { Breast can- } \\
\text { cer survivors } \\
\text { and others } \\
\mathrm{N}=462 / \text { mean } \\
\text { age }=55.9 \\
\text { years } / 79.9 \% \\
\text { female }\end{array}$ & $\begin{array}{c}\text { Online } \\
\text { workshop } \\
\text { (website) }\end{array}$ & $\begin{array}{l}\text { Two-arm RCT---KNW } \\
\text { intervention and } \\
\text { usual care } \\
\text { KNW: self-manage- } \\
\text { ment program }\end{array}$ & $\begin{array}{c}\text { PA } \\
\text { Diet } \\
\text { Smoking }\end{array}$ & 12 months & $\begin{array}{l}\text { Theory of } \\
\text { Planned } \\
\text { Behavior } \\
\text { Self-regula- } \\
\text { tion theory } \\
\text { I-Change } \\
\text { Model }\end{array}$ & $\begin{array}{l}\text { PA: } \\
\text { SQUASH } \\
\text { Diet: } \\
\text { Adapted } \\
\text { Dutch } \\
\text { Standard } \\
\text { Question- } \\
\text { naire on } \\
\text { Food Con- } \\
\text { sumption }\end{array}$ & $\begin{array}{c}\text { Increased moderate PA---sig- } \\
\text { nificant differences between } \\
\text { arms: } \\
\mathrm{B}=117.738, \mathrm{p}=.037, \mathrm{p} \\
\mathrm{fdr}=.148, \mathrm{~d}=-0.25, \mathrm{f}^{2}=.007 \\
\text { Short-term increase in vegeta- } \\
\text { ble intake } \\
\text { Complete cases: } \mathrm{B}=9.15 \\
\mathrm{p}=.027, \mathrm{p} \text { fdr }=.148, \mathrm{~d}=-0.37, \\
\mathrm{f}^{2}=-.013 ; \text { Intention-to-treat: } \\
\mathrm{B}=9.57, \mathrm{p}=.023, \mathrm{p} \mathrm{fdr}=.160 \\
\text { Attrition } \\
\text { Overall: } 11.5 \% \text { at } 6 \text { months : } \\
17.5 \% \text { at } 12 \text { months follow-up } \\
\text { KNW: } 18.6 \% \text { at } 6 \text { months; } \\
27 \% \text { at } 12 \text { months } \\
\text { Control: } 4.3 \% \text { at } 6 \text { months; } \\
8.2 \% \text { at } 12 \text { months }\end{array}$ \\
\hline $\begin{array}{l}\text { Kuijpers } \\
\text { [18] } \\
\text { Nether- } \\
\text { lands }\end{array}$ & $\begin{array}{c}\text { Breast cancer } \\
\text { survivors } \\
\mathrm{N}=92 / \text { mean } \\
\text { age }=49.5 / \\
100 \% \text { female }\end{array}$ & Website & $\begin{array}{l}\text { One-arm, pre/post- } \\
\text { test design } \\
\text { MijnAVL: website } \\
\text { with access to } \\
\text { medical records, } \\
\text { personalized healthy } \\
\text { lifestyle materials, } \\
\text { PA feedback and } \\
\text { support }\end{array}$ & PA & 16 weeks & $\begin{array}{c}\text { Social } \\
\text { Cognitive } \\
\text { Theory }\end{array}$ & PA: IPAQ & $\begin{array}{c}\text { No significant change in PA } \\
\text { Baseline: } 2793 \text { (MET-min/ } \\
\text { week) } \\
\text { Follow-up: } 3724.2 \text { (MET-min/ } \\
\text { week) } \\
\text { Attrition } \\
0 \% \text { at follow-up }\end{array}$ \\
\hline $\begin{array}{l}\text { Lynch, } \\
2016[19] \\
\text { USA }\end{array}$ & $\begin{array}{c}\text { Overweight } \\
\text { testicular } \\
\text { and breast } \\
\text { cancer survi- } \\
\text { vors } \\
\mathrm{N}=46 / \mathrm{mean} \\
\text { age }=39 / 65 \% \\
\text { female } / 98 \% \\
\text { White }\end{array}$ & $\begin{array}{l}\text { Website } \\
\text { and email }\end{array}$ & $\begin{array}{l}\text { One-arm, pre/post- } \\
\text { test design } \\
\text { Intervention: Com- } \\
\text { mercially available } \\
\text { website (Lean } \\
\text { Eating)3 daily com- } \\
\text { ponents: exercise, } \\
\text { nutritional/behav- } \\
\text { ioral modification, } \\
\text { health lessons. Daily } \\
\text { email reminders to } \\
\text { log, coach assistant }\end{array}$ & $\begin{array}{l}\text { Weight } \\
\text { manage- } \\
\text { ment } \\
\text { Second- } \\
\text { ary: PA } \\
\text { Diet }\end{array}$ & $\begin{array}{c}12 \text { months } \\
\text { with mea- } \\
\text { surements at } \\
0,6 \text {, and } 12 \\
\text { months }\end{array}$ & $\begin{array}{c}\text { Social } \\
\text { Cognitive } \\
\text { Theory }\end{array}$ & $\begin{array}{l}\text { Study } \\
\text { generated } \\
\text { question- } \\
\text { naire }\end{array}$ & $\begin{array}{c}\text { No change in PA } \\
\text { Body fat } \%: \\
\text { Baseline: } 26.6 \%(4.7) \\
6 \text { months: } 24.4 \%(5.3) \text {, } \\
\text { p= } 0.0004 \\
12 \text { months: } 22.2 \%(4.9) \text {, } \\
\text { p=0.002 } \\
\text { Attrition } \\
41 \% \text { at } 6 \text { months; } 51 \% \text { at } \\
\text { month } 12\end{array}$ \\
\hline $\begin{array}{c}\text { Pusz- } \\
\text { kiewicz, } \\
2016[20] \\
\text { United } \\
\text { Kingdom }\end{array}$ & $\begin{array}{c}\text { Breast, } \\
\text { prostate, and } \\
\text { colorectal } \\
\text { cancer survi- } \\
\text { vors } \\
\mathrm{N}=11 / \text { mean } \\
\text { age }=45 / \\
82 \% \text { female/ } \\
82 \% \text { White } \\
\text { British }\end{array}$ & $\begin{array}{l}\text { Mobile ap- } \\
\text { plication }\end{array}$ & $\begin{array}{l}\text { One-arm, pre/post- } \\
\text { test design } \\
\text { GAINFitness: Com- } \\
\text { mercially available } \\
\text { PA mobile applica- } \\
\text { tion; goal setting, PA } \\
\text { plans }\end{array}$ & $\begin{array}{l}\text { PA } \\
\text { Second- } \\
\text { ary: } \\
\text { Weight } \\
\text { mgmt. }\end{array}$ & 6 weeks & N/A & PA: GLTEQ & $\begin{array}{c}\text { Increased PA } \\
\text { significant increase in stren- } \\
\text { uous PA between baseline } \\
\text { (median=40, IQR=105) and } \\
\text { follow-up (median=120, } \\
\text { IQR=150), ( }=-2.80, P=.002) \\
\text { Mild PA: } \\
\text { baseline (median=150, } \\
\text { IQR=90) and follow-up (me- } \\
\text { dian=80, IQR=120), ( }=-2.21 \text {, } \\
\mathrm{P}=.031 \\
\text { No change in BMI: } \\
23.9(5.2) \text { vs } 23.4(5.0) \\
\text { p=0.828 } \\
\text { Attrition } \\
0 \% \text { at follow-up }\end{array}$ \\
\hline
\end{tabular}




\begin{tabular}{|c|c|c|c|c|c|c|c|c|}
\hline $\begin{array}{c}\text { Golsteijn, } \\
2017 \text { [21] } \\
\text { Golsteijn, } \\
2018 \text { [22] } \\
\text { Nether- } \\
\text { lands }\end{array}$ & $\begin{array}{c}\text { Colorectal or } \\
\text { prostate can- } \\
\text { cer survivors } \\
\mathrm{N}=510 / \\
\text { mean age }= \\
66 \text { years } / \\
13 \% \text { female }\end{array}$ & Website & $\begin{array}{l}\text { 2-arm RCT: OncoAct- } \\
\text { ive vs usual care } \\
\text { Computer-tailored } \\
\text { PA intervention with } \\
\text { interactive website } \\
\text { and print materials } \\
\text { Provides feedback, } \\
\text { goal setting }\end{array}$ & PA & $\begin{array}{l}16 \text { weeks } \\
2 \text { month } \\
\text { follow-up post } \\
\text { intervention }\end{array}$ & $\begin{array}{l}\text { I-Change } \\
\text { Model } \\
\text { Social } \\
\text { Cognitive } \\
\text { Theory } \\
\text { Transthe- } \\
\text { oretical } \\
\text { Model } \\
\text { Health Be- } \\
\text { lief Model } \\
\text { Precaution } \\
\text { Adoption } \\
\text { Process } \\
\text { Model } \\
\text { Goal setting } \\
\text { theories } \\
\text { Health ac- } \\
\text { tion process } \\
\text { approach } \\
\text { Theories of } \\
\text { self-regula- } \\
\text { tion }\end{array}$ & $\begin{array}{c}\text { PA: } \\
\text { ActiGraph } \\
\text { Acceler- } \\
\text { ometer, } \\
\text { SQUASH }\end{array}$ & $\begin{array}{c}\text { Increased PA } \\
\text { Acti Graph } \\
\text { Baseline-Onco Active vs usual } \\
\text { care: } \\
271 \text { (211) vs. } 293 \text { (230), } \\
\text { p=0.30 } \\
\text { Follow-up-Onco Active vs } \\
\text { usual care: } \\
331 \text { (234) vs. } 301 \text { (219), } \\
\text { p-0.006 } \\
\text { SQUASH } \\
\text { Baseline-Onco Active vs } \\
\text { usual care: } \\
780 \text { (721) vs. } 873 \text { (764), } \\
\text { p=0.29 } \\
\text { Follow-up-Onco Active vs } \\
\text { usual care: } \\
1145 \text { (883) vs. } 213 \text { (943), } \\
\text { p<0.001 } \\
\text { Attrition } \\
\text { Overall: } 4.4 \% \text { at } 16 \text { weeks; } \\
7.3 \% \text { at } 2 \text { month follow up; } \\
\text { Intervention: } \\
6.0 \% \text { at } 16 \text { weeks } \\
9.6 \% \text { at } 2 \text { month follow-up } \\
\text { post intervention } \\
\text { Control: } \\
2.6 \% \text { at } 16 \text { weeks } \\
4.8 \% \text { at } 2 \text { month follow-up }\end{array}$ \\
\hline $\begin{array}{c}\text { Paxton, } \\
2017 \text { [23] } \\
\text { USA }\end{array}$ & $\begin{array}{l}\text { Breast cancer } \\
\text { survivors } \\
\mathrm{N}=71 / \text { mean } \\
\text { age }=52 \\
\text { years } / 100 \% \\
\text { female/ } \\
\text { 83\% African } \\
\text { American }\end{array}$ & $\begin{array}{l}\text { Website \& } \\
\text { Email }\end{array}$ & $\begin{array}{c}\text { Two-arm RCT } \\
\text { Parallel-group feasi- } \\
\text { bility study } \\
\text { ALIVE: Randomized } \\
\text { to physical activity } \\
\text { or diet email-based } \\
\text { intervention }\end{array}$ & $\begin{array}{c}\text { PA } \\
\text { Diet }\end{array}$ & 12 weeks & $\begin{array}{c}\text { Social } \\
\text { Cognitive } \\
\text { Theory } \\
\text { TTM } \\
\text { Goal-setting } \\
\text { theory } \\
\text { Social mar- } \\
\text { keting }\end{array}$ & $\begin{array}{l}\text { PA: study } \\
\text { generated } \\
\text { question- } \\
\text { naire } \\
\text { Diet: study } \\
\text { generated } \\
\text { question- } \\
\text { naire }\end{array}$ & $\begin{array}{c}\text { Increased PA } \\
\text { PA arm: }+165 \text { mins/week (68) } \\
\text { vs Diet arm: }+75 \text { mins/week } \\
\text { (62), } \mathrm{p}<0.001 \\
\text { Diet---saturated fat } \\
\text { PA arm: }-1.0 \text { grams/day }(1.3) \\
\text { vs Diet arm: }-0.8(1.2), \mathrm{p}=0.46 \\
\text { Diet---vegetables and fruits } \\
\text { PA arm: }+0.6 \text { cups/day }(0.3) \mathrm{vs} \\
\text { Diet arm: }+1.0(0.3), \mathrm{p}=0.35 \\
\text { Attrition } \\
\text { Overall: } 38 \% \text { at } 3 \text { months } \\
\text { PA arm: } 41.2 \% \text { at } 12 \text { weeks } \\
\text { Diet arm: } 35.1 \% \text { at } 12 \text { weeks }\end{array}$ \\
\hline $\begin{array}{c}\text { Short, } \\
2017 \text { [24] } \\
\text { Australia }\end{array}$ & $\begin{array}{c}\text { Breast cancer } \\
\text { survivors } \\
\mathrm{N}=492 / \\
\text { mean age }= \\
55 \text { years / } \\
100 \% \text { female }\end{array}$ & Website & $\begin{array}{l}\text { 3-arm RCT } \\
\text { Three-arms: (1) a } \\
\text { computer-tailored } \\
\text { three-module inter- } \\
\text { vention delivered } \\
\text { monthly; (2) a } \\
\text { computer-tailored } \\
\text { three-module inter- } \\
\text { vention delivered } \\
\text { weekly (over the } \\
\text { first } 3 \text { weeks) or (3) } \\
\text { a computer-tailored } \\
\text { single module inter- } \\
\text { vention }\end{array}$ & PA & 12 weeks & $\begin{array}{c}\text { Social } \\
\text { Cognitive } \\
\text { Theory }\end{array}$ & PA: GLTEQ & $\begin{array}{c}\text { Increased PA in each arm from } \\
\text { baseline to follow-up: } \\
\text { Arm 1: } 90.08 \text { mins/week } \\
\text { (106.63) vs } 216.99 \text { (219.99), } \\
\text { p }<0.05 \\
\text { Arm 2: } 97.17 \text { mins/week } \\
\text { (124.10) vs } 186.08 \text { (157.89), } \\
\text { p }<0.05 \\
\text { Arm 3: } 96.15 \text { mins/week } \\
\text { (119.63) vs } 186.05 \text { (172.56), } \\
\text { p }<0.05 \\
\text { Attrition } \\
\text { Overall: } 68 \% \text { at } 12 \text { weeks } \\
\text { follow-up } \\
\text { Arm 1: } 71 \% \\
\text { Arm } 2: 73 \% \\
\text { Arm 3: } 61 \%\end{array}$ \\
\hline
\end{tabular}




\begin{tabular}{|c|c|c|c|c|c|c|c|c|}
\hline $\begin{array}{l}\text { Uhm, } \\
2017 \text { [25] } \\
\text { South } \\
\text { Korea }\end{array}$ & $\begin{array}{c}\text { Breast cancer } \\
\text { survivors } \\
\mathrm{N}=356 / \text { mean } \\
\text { age }=50.3 / \\
100 \% \text { female }\end{array}$ & $\begin{array}{l}\text { Mobile ap- } \\
\text { plication }\end{array}$ & $\begin{array}{l}\text { Quasi two-arm RCT } \\
\text { Smart After Care: } \\
\text { Access to mobile } \\
\text { PA application and } \\
\text { pedometer } \\
\text { Control: Treatment } \\
\text { as usual-given PA } \\
\text { brochure }\end{array}$ & $\begin{array}{l}\text { PA } \\
\text { QoL } \\
\text { Second- } \\
\text { ary: } \\
\text { BMI } \\
\text { (weight } \\
\text { manage- } \\
\text { ment }\end{array}$ & 12 weeks & N/A & $\begin{array}{l}\text { PA: IP- } \\
\text { AQ-SF }\end{array}$ & $\begin{array}{c}\text { Increased PA (METs) } \\
\text { Intervention---Baseline } \\
2050.6 \pm 2182.2 \text { vs Follow-up } \\
3026.9 \pm 2489.5, \mathrm{p}<0.05 \\
\text { Control---Baseline } 2091.5 \pm \\
1811.2 \text { vs Follow-up } 2560.4 \pm \\
2354.9, \mathrm{p}<0.05 \\
\text { No change in BMI: } \\
\text { Intervention--Baseline } 23.3 \pm \\
3.1 \text { vs Follow-up } 23.3 \pm 3.1 \\
\text { Control---Baseline } 23.3 \pm 3.3 \\
\text { vs Follow-up } 23.3 . \pm 3.4 \\
\text { Attrition } \\
\text { Overall: } \\
\text { 3.9\% at } 6 \text {-weeks } 4.8 \% \text { at } \\
12 \text {-weeks } \\
\text { Intervention: } \\
5.6 \% \text { at } 6 \text { weeks } \\
6.7 \% \text { at } 12 \text { weeks } \\
\text { Control: } \\
2.3 \% \text { at } 6 \text { weeks } \\
2.8 \% \text { at } 12 \text { weeks }\end{array}$ \\
\hline $\begin{array}{l}\text { Trinh, } \\
2018[26] \\
\text { Trinh, } \\
2018[27] \\
\text { Canada }\end{array}$ & $\begin{array}{l}\text { Prostate can- } \\
\text { cer survivors } \\
\mathrm{N}=46 / \text { mean } \\
\text { age }=73.2 \\
\text { years } / 100 \% \\
\text { male } / 80.4 \% \\
\text { White }\end{array}$ & $\begin{array}{l}\text { Mobile ap- } \\
\text { plication }\end{array}$ & $\begin{array}{l}\text { One-arm, pre-/post- } \\
\text { test design } \\
\text { Feasibility study } \\
\text { Rise Tx: Access to } \\
\text { application aimed at } \\
\text { increasing PA }\end{array}$ & $\begin{array}{l}\text { Second- } \\
\text { ary: } \\
\text { PA }\end{array}$ & $\begin{array}{l}12 \text { weeks } \\
3 \text {-month } \\
\text { follow-up }\end{array}$ & $\mathrm{N} / \mathrm{A}$ & $\begin{array}{l}\text { PA: Acti } \\
\text { Graph } \\
\text { Acceler- } \\
\text { ometer }\end{array}$ & $\begin{array}{c}\text { Increased PA significantly } \\
\text { from baseline to } 12 \text { weeks } \\
\text { post } \\
\text { Baseline: } \\
93.1 \text { mins/week (14.5) } \\
12 \text { weeks: } \\
137.1 \text { mins/week (22.8), } \\
\text { p =.010 } \\
\text { 3-months post-intervention: } \\
122.1 \text { (23.9) mins/week, } \\
\text { p =.18 } \\
\text { Attrition } \\
4.7 \% \text { at follow-up }\end{array}$ \\
\hline
\end{tabular}

Abbreviations: BMI: Body Mass Index; GLTEQ: Godin Leisure-Time Exercise Questionnaire; IPAQ: International Physical Activity Questionnaires; IPAQ-SF: International Physical Activity Questionnaires short form PA: Physical activity; METs: Metabolic equivalents; N/A: Not applicable; PAR: Seven-Day Physical Activity Recall; QoL: Quality of Life; RCT: Randomized control trial; SQUASH: Short Questionnaire to Assess Health-Enhancing

*Weight was measured using a scale.

The RCTs had a variety of control conditions. In the two-arm RCTs, the control groups were either wait-listed [21,23,34,35], received self-help materials related to the intervention (e.g., physical activity information) [20,28], usual care [22,36,38], or health information not directly related to the intervention [25]. The three-arm RCT assessed the relative efficacy of three different website delivery schedules (three intervention modules monthly vs. three intervention modules weekly vs. one intervention module) [37]. Intervention duration ranged from 1-12 months, but most programs $(\mathrm{N}=15)$ were short-term (6-12 weeks) [20-29,34]. Only four studies $[23,24,34,36]$ had follow-up post-intervention. Two studies intervened for 12 -weeks and assessed outcomes at 12 weeks as well as three months post-intervention [23,24]. Other studies followed-up six months after intervening for six weeks [34] and followed up two months after a 16-week intervention [36]. The overall percentage of dropouts ranged between $0 \%$ to $68 \%$ with most $(n=16)$ studies reporting attrition rates $\leq 30 \%$. Intervention targets and components. All of the included web- based lifestyle studies intervened on physical activity as either a primary [20-23,25-28,31-38] or secondary objective [24,29,30]. Studies also targeted diet $(\mathrm{N}=7)[26,28-31,34,35]$ and weight management $(\mathrm{N}=5)[23,29,30,32,38]$, as well as smoking $[31,35]$, depression [34], fatigue [34], and quality of life [38]. Across the studies, the Internet was used in a variety of ways to deliver healthy lifestyle interventions to cancer survivors. Intervention modalities included program websites ( $\mathrm{N}=12$ ) $[20,22,23,25,26,28-31,33-37]$, mobile applications $[24,27,29,32,38]$, email $[21,22,25,26,31]$, and Facebook [20]. Some studies $(\mathrm{N}=7)$ used more than one Internetbased method [20,22,25,26,29-31] by combining a website with social media (i.e., Facebook) [20], a mobile application [29], or email [22,25,26,30,31].

Only one intervention relied solely on email for intervention delivery [21]. Intervention emails included healthy lifestyle tips $[21,26]$, feedback based on emailed responses to open-ended questions [21], healthy lifestyle goal recommendations [26], 
healthy lifestyle educational modules [31] and healthy lifestyle behavior reminders [26,30] (e.g., logging weight $[26,30]$ and physical activity [26]). Common website features/components included goal setting [20,22,23,25,28,29,33-37], self-monitoring (physical activity, weight, or diet) [20,22,25,28,29,33,34,37], and tailored feedback $[20,28,29,31,33,35-37]$. Websites also provided educational healthy lifestyle modules [22,31,37] (e.g., benefits of exercise, exercise safety, relapse prevention, and building a support network). Mobile applications had similar features as intervention websites as they offered goal setting [27,29,32], selfmonitoring(physical activity, weight, or diet) $[24,27,29]$, social networking (e.g., discussion forum where cancer survivors can communicate with other survivors) [27], physical activity plans [32], and tailored feedback [27]. Three studies used commercially available healthy lifestyle websites and applications (e.g., LoseIt [29], Lean Eating [30], and GAIN Fitness [32]), which were not explicitly designed for cancer survivors [29,30,32].

Measuring behavior change: Diet quality was assessed using the Dutch Standard Questionnaire on Food Consumption [35], a study generated questionnaire with 35 commonly consumed foods identified as significant contributors to the intake of added sugars, fruits and vegetables, and saturated and trans fats in the National Health and Nutrition Examination Survey [26], the Block Food Frequency Questionnaire [34], three- day dietary recall that assessed diet with the Diet Quality Index (DQI) [28], and the Loselt application [29]. Studies also relied on self-report to measure changes in physical activity except for three studies where accelerometers (e.g., Acti Graph [24,36]) and pedometers [24] (e.g., New Lifestyles [23]) Jawbone [24]) were used. Selfreport changes in physical activity were measured with the Godin Leisure-Time Exercise Questionnaire (GLTEQ) [20,22,32,34,37], the Seven-Day Physical Activity Recall (PAR) [21,25,30], the Short Questionnaire to Assess Health-Enhancing (SQUASH) Physical Activity [35,36], the LoseIt application [29], and the International Physical Activity Questionnaires (IPAQ - both the original and short forms) $[33,38]$, and study generated questionnaires [2628,31]. Of the study created physical activity instruments, only two were adapted from existing measures (e.g., Behavioral Risk Factors Surveillance System [31] and Cross-Cultural Activity Participation Study [CAPS] [26]). Participants were weighed using scales $[23,29,30,32,38]$.

Behavior change outcomes: All included studies targeted physical activity and most $(\mathrm{N}=15)$ reported increases in physical activity [20-22,24-29,32,34-38]. Increases in physical activity ranged from 31 to 126 minutes/week (for studies that reported mins/week) [20,22-26,32,34-37]. Other studies indicated an increase in metabolic equivalents (METs) [38] or the frequency of participants being physically active $[21,27,28]$ (e.g., number of people engaging in $\geq 150$ minutes physical activity per week or the number of days of physical activity). Of the studies that reported an increase in physical activity, one study found that both the intervention and self-help comparison group increased self-reported physical activity [20]. Five studies examined weight management among adult cancer survivors [23,29,30,32,38] with three reporting no weight loss $[23,32,38]$, one reporting statistically significant weight improvements at six and 12 months [30], and another finding clinically significant weight loss at four weeks [29]. Of seven studies targeting diet composition [26,28$31,34,35]$, three found improvements in vegetable and fruit intake $[26,28,35]$, fiber [26], saturated fat [26], and trans-fat [26]. The other four studies found no change in diet [29-31,34].

Theoretical framework: The majority of the studies (15-outof-19) reported using theoretical framework(s) to guide their intervention, which included: Social Cognitive Theory (SCT) $[20,21,23,25,26,30,33,37,39]$, the Transtheoretical Model (TTM) $[25,26,28,36]$, the Theory of Planned Behavior (TPB) [22,35], the Theory of Reasoned Action (TRA) [31], Goal Setting Theory $[26,27,36]$, Social Marketing Theory [26], Self-regulation Theory [35], the I-Change Model [35,36], the Health Belief Model (HBM) [36], the Precaution Adoption Process Model (PAPM) [36], the Health Action Process Approach [36], and Theories of Selfregulation [36]. Eleven studies [20-22,27,28,30,31,33,37,39] used one theoretical framework, while others $(\mathrm{N}=4)[25,26,35,36]$ used multiple theories/models. SCT was the most commonly cited model in this review $(\mathrm{N}=10)[20,21,23,25,26,30,33,37,39]$, and TTM was used in four studies $[25,26,28,36]$. Most studies $(\mathrm{N}=10)$ $[20,21,25,26,28,30,33,35-37]$ provided detailed description of how theory was incorporated in intervention development.

\section{Discussion}

Internet-based approaches to promote behavioral change in cancer survivors appear promising. All the included studies targeted physical activity, and most were associated with increases in physical activity among cancer survivors. Few interventions targeted weight management and diet quality. However, an estimated $20 \%$ of cancer cases and $30 \%$ of cancer deaths are attributed to the combined effects of an unhealthy diet, excess body weight, and physical inactivity $[40,41]$. Lifestyle behaviors are modifiable cancer risk factors that can be addressed to improve the quality and quantity of life among cancer survivors [42]. Therefore, future interventions should target a combination of physical activity, weight management, and diet quality as cancer survivors often require multiple behavior changes to improve their quality of life $[43,44]$. Behavior change theory has been shown to improve the effectiveness of health behavior change interventions [45-47]. Yet, the descriptions of the theoretical framework used for intervention development varied in detail. Most studies explained how the theory was used for program development (e.g., theoretical constructs were targeted by specific intervention strategies and incorporated into the assessment), but some did not. Future web-delivered lifestyle interventions for cancer survivors should continue using theoretical frameworks for program development and explicitly report how theory is integrated. 
Studies included in this review generally focused on shortterm improvements in health behavior and tended to forgo following up post-intervention to see if these changes were maintained. While short-term change is favorable, long-term health behavior modifications have lasting health implications (e.g., reduced cancer and recurrence risk). Maintaining healthy lifestyle behaviors is often a challenge as people return to unhealthy lifestyle behaviors [48]. Nevertheless, lengthier interventions and follow-up periods are needed to determine whether web-delivered lifestyle interventions facilitate long-term behavior change. Small sample sizes also were a major limitation of many of the studies that were reviewed. Among the RCTs, many did not appear to have appropriate sample sizes for statistical power. Quasi-RCTs and single-arm pre-/post-test study designs were included in this review as they highlight ongoing research in the field. However, these studies were also typically underpowered. Therefore, future research should include larger sample sizes for greater statistical power to detect intervention effects, especially among segments of cancer survivors.

Lifestyle interventions for cancer survivors have been predominately for cancer-specific populations [16]. A prior review of broad-reach (comprised of telephone, print, and web intervention) lifestyle interventions for cancer survivors found that most programs were conducted with single cancer populations (i.e., exclusively for breast or prostate cancer survivors [16]. However, unhealthy lifestyle behaviors are present and problematic across all cancer types and require appropriate interventions [1]. Encouragingly, this review found that web-delivered lifestyle intervention studies included more "diagnosis diverse" (cancer survivors of more than cancer-type) populations than tend to be reported for face-to-face clinicbased interventions. Of the 19 studies included in the review, 12 studies included diagnosis diverse populations with three studies targeting two populations of cancer survivors (e.g., breast and endometrial; colorectal and prostate; and testicular and breast) and two studies targeting three populations of cancer survivors (e.g., breast, colorectal, and prostate). Therefore, only seven studies targeted single diagnosis populations (e.g., breast and prostate cancer survivors). Despite cancer diagnosis diversity among participants, populations were still predominately female. For example, Puszkiewicz et al. included breast, prostate, and colorectal cancer survivors and the population was $82 \%$ female. Therefore, future studies should target diagnosis diverse male cancer survivors. Studies with diagnosis diverse populations and male representation increase the generalizability of findings among cancer survivor groups, as does minority representation.

There is still a lack of participation from ethnic and racial minorities in healthy lifestyle cancer research. Several studies $(\mathrm{N}=6)$ did not report the racial/ethnic distribution of their study samples and of the studies that did samples were predominately White, except for one study among African American breast cancer survivors [26]. African Americans are especially vulnerable to poor health outcomes and are disproportionately affected by many health conditions associated with poor diet quality, excess body weight, and physical inactivity [49]. More studies should specifically target racial and ethnic minorities, especially African Americans, to provide much-needed interventions and eliminate related cancer disparities.

\section{Conclusion}

Web-delivered lifestyle interventions provide a means for delivering health behavior change programs to meet the growing needs of cancer survivors. Overall, the literature suggests that webdelivered lifestyle interventions have the potential to promote healthy lifestyle behavior among cancer survivors, especially increasing physical activity. However, future lifestyle interventions should address methodological issues outlined in this article (e.g., multiple behavior change, targeting minority and male survivors, post-intervention follow-up periods, and larger sample sizes).

\section{Acknowledgments}

This research was supported by the American Cancer Society (CRP-14-111-01-CPPB), and the National Cancer Institute Cancer Prevention and Control Training Program (T32-CA047888).

\section{References}

1. (2019) Definitions, Statistics and Graph NCI.

2. (2018) American Cancer Society. Cancer Facts \& Figures.

3. (2017) Understanding Cancer.

4. Vijayvergia N, Denlinger CS (2015) Lifestyle Factors in Cancer Survivorship: Where We Are and Where We Are Headed. J Pers Med 5(3): 243-263.

5. Berglund G, Christina Bolund, Ulla-Lena, Gustafsson, Per-Olow Sjödén (1994) A randomized study of a rehabilitation program for cancer patients: the 'starting again' group. Psycho-Oncology 3(2): 109-120.

6. Burnham TR, Wilcox A, (2002) Effects of exercise on physiological and psychological variables in cancer survivors. Med Sci Sports Exerc 34(12): 1863-1867.

7. Demark-Wahnefried W, Clipp EC, Morey MC, Pieper CF, Sloane R, et al. (2006) Lifestyle intervention development study to improve physical function in older adults with cancer: outcomes from Project LEAD. J Clin Oncol 24(21): 3465-3473.

8. Stull VB, Snyder DC, Demark-Wahnefried W (2007) Lifestyle interventions in cancer survivors: designing programs that meet the needs of this vulnerable and growing population. J Nutr 137(1 Suppl): 243s-248s.

9. Joseph RP, Durant NH, Benitez TJ, Pekmezi DW (2014) Internet-Based Physical Activity Interventions. Am J Lifestyle Med 8(1): 42-68.

10. Van den Berg MH, Schoones JW, Vliet Vlieland TP (2007) Internet-based physical activity interventions: a systematic review of the literature. J Med Internet Res 9(3): e26.

11. Vandelanotte C, Morwenna Kirwan, Amanda Rebar, Stephanie Alley, Camille Short, et al. (2014) Examining the use of evidence-based and social media supported tools in freely accessible physical activity 
intervention websites. International Journal of Behavioral Nutrition and Physical Activity 11(1): 105.

12. Ritterband LM, Thorndike FP, Cox DJ, Kovatchev BP, Gonder-Frederick LA (2009) A Behavior Change Model for Internet Interventions. Ann Behav Med 38(1): 18-27.

13. Wantland DJ (2004) The Effectiveness of Web-Based vs. Non-WebBased Interventions: A Meta-Analysis of Behavioral Change Outcomes. J Med Internet Res 6(4): e40.

14. Mattsson S, Olsson EMG, Johansson B, Carlsson M (2017) HealthRelated Internet Use in People with Cancer: Results from a CrossSectional Study in Two Outpatient Clinics in Sweden. J Med Internet Res 19(5): e163-e163.

15. Holmes MM (2019) Why People Living with and Beyond Cancer Use the Internet. Integrative cancer therapies 18: 15347354198298301534735419829830.

16. Goode A, Lawler SP, Brakenridge CL, Reeves MM, Eakin EG (2015) Telephone, print, and Web-based interventions for physical activity, diet, and weight control among cancer survivors: a systematic review. J Cancer S 9(4): 660-682.

17. Haberlin C, O Dwyer T, Mockler D, Moran J, O’Donnell DM, et al. (2018) The use of eHealth to promote physical activity in cancer survivors: a systematic review. Support Care Cancer 26(10): 3323-3336.

18. Roberts A, Fisher A, Smith L, Heinrich M, Potts HWW (2017) Digital health behaviour change interventions targeting physical activity and diet in cancer survivors: a systematic review and meta-analysis. J Cancer Surviv 11(6): 704-719.

19. Moher, D Liberati A, Tetzlaff J, Altman DG (2009) Preferred reporting items for systematic reviews and meta-analyses: the PRISMA statement. Ann Intern Med 151(4): 264-269.

20. Valle CG (2013) A randomized trial of a Facebook-based physical activity intervention for young adult cancer survivors. Journal of cancer survivorship: research and practice 7(3): 355-368.

21. Hatchett A, Hallam JS, Ford MA (2013) Evaluation of a social cognitive theory-based email intervention designed to influence the physical activity of survivors of breast cancer. Psychooncology 22(4): 829-836.

22. Forbes CC, Blanchard CM, Mummery WK, Courneya KS (2015) Feasibility and Preliminary Efficacy of an Online Intervention to Increase Physical Activity in Nova Scotian Cancer Survivors: A Randomized Controlled Trial. JMIR cancer 1(2): e12.

23. Frensham LJ, Parfitt G, Dollman J (2018) Effect of a 12-Week Online Walking Intervention on Health and Quality of Life in Cancer Survivors: A Quasi-Randomized Controlled Trial. Int J Environ Res Public Health 15(10).

24. Trinh L, Arbour-Nicitopoulos KP, Sabiston CM, Berry SR, Loblaw A, et al (2018) Rise Tx: testing the feasibility of a web application for reducing sedentary behavior among prostate cancer survivors receiving androgen deprivation therapy. The international journal of behavioral nutrition and physical activity 15(1): 49-49.

25. Rabin C, Dunsiger S, Ness KK, Marcus BH (2011) Internet-Based Physical Activity Intervention Targeting Young Adult Cancer Survivors. J Adolesc Young Adult Oncol 1(4): 188-194.

26. Paxton, RJ, Hajek R, Newcomb P, Dobhal M, Borra S, et al. (2017) A Lifestyle Intervention via Email in Minority Breast Cancer Survivors: Randomized Parallel-Group Feasibility Study. JMIR Cancer 3(2): e13.

27. Hong YA, Goldberg D, Ory MG, Towne SD, Forjuoh SN, et al. (2015) Efficacy of a Mobile-Enabled Web App (i Can Fit) in Promoting Physical Activity Among Older Cancer Survivors: A Pilot Study. JMIR Cancer 1(1): e7.
28. Lee MK, Yun YH, Park HA, Lee ES, Jung KH, et al. (2014) A Web-based self-management exercise and diet intervention for breast cancer survivors: Pilot randomized controlled trial. International Journal of Nursing Studies 51(12): 1557-1567.

29. McCarroll ML, Armbruster S, Pohle-Krauza RJ, Lyzen AM, Min S, et al. (2015) Feasibility of a lifestyle intervention for overweight/obese endometrial and breast cancer survivors using an interactive mobile application. Gynecol Oncol 137(3): 508-515.

30. Lynch SM, Stricker CT, Brown JC, Berardi JM, Vaughn D, et al. (2017) Evaluation of a web-based weight loss intervention in overweight cancer survivors aged 50 years and younger. Obesity science \& practice 3(1): 83-94.

31. Berg CJ, Stratton E, Giblin J, Esiashvili N, Mertens A (2014) Pilot results of an online intervention targeting health promoting behaviors among young adult cancer survivors, Psychooncology 23(10): 1196-1199.

32. Puszkiewicz P, Roberts AL, Smith L, Wardle J, Fisher A (2016) Assessment of Cancer Survivors' Experiences of Using a Publicly Available Physical Activity Mobile Application. JMIR Cancer 2(1): e7.

33. Kuijpers W, Groen WG, Oldenburg HS, Wouters MW, Aaronson NK, et al. (2016) eHealth for Breast Cancer Survivors: Use, Feasibility and Impact of an Interactive Portal. JMIR Cancer 2(1): e3.

34. Bantum EO, Albright CL, White KK, Berenberg JL, Layi G, et al. (2014) Surviving and thriving with cancer using a Web-based health behavior change intervention: randomized controlled trial. J Med Internet Res 16(2): e54.

35. Kanera IM, Bolman CA, Willems RA, Mesters I, Lechner L, et al. (2016) Lifestyle-related effects of the web-based Kanker Nazorg Wijzer (Cancer Aftercare Guide) intervention for cancer survivors: a randomized controlled trial. J Cancer Surviv 10(5): 883-897.

36. Golsteijn RHJ, Bolman C, Volders E, Peels DA, de Vries H, et al. (2018) Short-term efficacy of a computer-tailored physical activity intervention for prostate and colorectal cancer patients and survivors: a randomized controlled trial. The international journal of behavioral nutrition and physical activity 15(1): 106.

37. Short C, Rebar A, James EL, Duncan MJ, Courneya KS, et al. (2017) How do different delivery schedules of tailored web-based physical activity advice for breast cancer survivors influence intervention use and efficacy? J Cancer Surviv 11(1): 80-91.

38. Uhm KE, Yoo JS, Chung SH, Lee JD, Leel, et al. (2017) Effects of exercise intervention in breast cancer patients: is mobile health (mHealth) with pedometer more effective than conventional program using brochure? Breast Cancer Res Treat 161(3): 443-452.

39. McCarroll ML, Armbruster S, Frasure HE, Gothard MD, Gil KM, et al. (2014) Self-efficacy, quality of life, and weight loss in overweight/obese endometrial cancer survivors (SUCCEED): a randomized controlled trial. Gynecol Oncol 132(2): 397-402.

40. Islami F, Goding Sauer A, Miller KD, Siegel RL, Fedewa SA, et al. (2018) Proportion and number of cancer cases and deaths attributable to potentially modifiable risk factors in the United States. CA: A Cancer J Clin 68(1): 31-54.

41. Kohler LN, Garcia DO, Harris RB, Oren E, Roe DJ, et al. (2016) Adherence to Diet and Physical Activity Cancer Prevention Guidelines and Cancer Outcomes: A Systematic Review. Cancer Epidemiol Biomarkers Prev 25(7): 1018-1028.

42. Amireault S, Fong AJ, Sabiston CM (2016) Promoting Healthy Eating and Physical Activity Behaviors: A Systematic Review of Multiple Health Behavior Change Interventions Among Cancer Survivors. American Journal of Lifestyle Medicine 12(3): 184-199.

43. Prochaska JJ, Spring B, Nigg CR (2008) Multiple health behavior change 
research: an introduction and overview. Prev Med 46(3): 181-188.

44. Spring B (2015) Fostering multiple healthy lifestyle behaviors for primary prevention of cancer. Am Psychol 70(2): 75-90.

45. Davis R (2015) Theories of behaviour and behaviour change across the social and behavioural sciences: a scoping review. Health Psychol Rev 9(3): 323-344.

46. Glanz K Bishop DB (2010) The role of behavioral science theory in development and implementation of public health interventions. Annu
Rev Public Health 31: 399-418.

47. Bluethmann SM, Bartholomew LK, Murphy CC, Vernon SW (2017) Use of Theory in Behavior Change Interventions. The official publication of the Society for Public Health Education, Health Educ Behav 44(2): 245-253.

48. Middleton KR, Anton SD, Perri MG (2013) Long-Term Adherence to Health Behavior Change. Am J Lifestyle Med 7(6): 395-404.

49. DeSantis CE, Miller KD, Goding Sauer A, Jemal A, Siegel RL (2019)

\section{Your next submission with Juniper Publishers will reach you the below assets}

- Quality Editorial service

- Swift Peer Review

- Reprints availability

- E-prints Service

- Manuscript Podcast for convenient understanding

- Global attainment for your research

- Manuscript accessibility in different formats

( Pdf, E-pub, Full Text, Audio)

- Unceasing customer service

Track the below URL for one-step submission https://juniperpublishers.com/online-submission.php 